Published in final edited form as:

J Clin Densitom. 2015 April ; 18(2): 145-149. doi:10.1016/j.jocd.2015.01.005.

\title{
DXA utilization between 2006 and 2012 in commercially insured younger postmenopausal women
}

\author{
Robert A. Overman, MPH ${ }^{\mathrm{a}, \mathrm{c}}$, Joel F. Farley, PhD ${ }^{\mathrm{a}}$, Jeffrey R. Curtis, MD, MPH, MS ${ }^{\mathrm{d}}$, Jie \\ Zhang, $\mathrm{PhD}^{\mathrm{e}}$, Margaret L. Gourlay, MD, $\mathbf{M P H}^{\mathrm{b}}$, and Chad L. Deal, $\mathrm{MD}^{\mathrm{C}}$ \\ aDivision of Pharmaceutical Outcomes and Policy, UNC Eshelman School of Pharmacy, Chapel \\ Hill, NC \\ bDepartment of Family Medicine, University of North Carolina, Chapel Hill, NC \\ 'Department of Rheumatology, Orthopedic and Rheumatologic Institute, Cleveland Clinic \\ Foundation, Cleveland, $\mathrm{OH}$ \\ dDivision of Clinical Immunology and Rheumatology, University of Alabama at Birmingham, \\ Birmingham, AL \\ eDepartment of Epidemiology, University of Alabama at Birmingham, Birmingham, AL
}

\section{Abstract}

Introduction-Reimbursement for dual energy x-ray absorptiometry (DXA) scans in the outpatient setting has declined significantly since 2006. Research through 2011 has suggested reimbursement reductions for DXA scans have corresponded with an overall decreased utilization of DXA. This study updates utilization estimates for DXAs through 2012 in patients with commercial insurance and compares DXA rates before and after reimbursement changes.

Methods-We evaluated DXA utilization for women age 50-64 from Marketscan Commercial Claims and Encounter database between January 2006 and December 2012 based on CPT codes. We estimated utilization rates per 1,000 person years. We also employed segmented regression analysis of monthly rates to evaluate the change in utilization rates after a proposed reimbursement reduction in July 2009.

\footnotetext{
(C) 2015 by the International Society for Clinical Densitometry. All rights reserved.

Corresponding Author: Robert A. Overman, MPH, University of North Carolina at Chapel Hill, UNC Eshelman School of Pharmacy, Division of Pharmaceutical Outcomes and Policy, Campus Box 7573, Chapel Hill, NC 27599-7573, Phone: 614-570-8041, Fax: 919-966-7557, overmar@unc.edu.

Publisher's Disclaimer: This is a PDF file of an unedited manuscript that has been accepted for publication. As a service to our customers we are providing this early version of the manuscript. The manuscript will undergo copyediting, typesetting, and review of the resulting proof before it is published in its final citable form. Please note that during the production process errors may be discovered which could affect the content, and all legal disclaimers that apply to the journal pertain.

Conflicts of Interest: JFF: consulting fees from Daiichi-Sankyo Pharmaceuticals; CLD: speaking \& consulting fees from Amgen and Eli Lily, JRC: research grants \& consulting from Amgen.

Authorship Contributions: All authors were involved in drafting the article or revising it critically for important intellectual content, and all authors approved the final version to be published. Robert Overman had full access to all of the data in the study and takes responsibility for the integrity of the data and the accuracy of the data analysis.

Study conception and design. Overman, Farley, Gourlay, Curtis, Deal.

Acquisition of data. Overman.

Analysis and interpretation of data. Overman, Farley, Curtis, Zhang, Gourlay, Deal.
} 
Results-In women aged 50-64; 451,656 DXAs were performed in 2006, a rate of 144 DXAs per 1,000 person years. This rate increased to 149 DXAs per 1,000 person years in 2009 before decreasing to 110 DXAs per 1,000 person years or 667,982 scans in 2012. DXA utilization increased by 2.24 per 1,000 person years until July 2009 then declined by 12.98 DXAs per 1,000 persons, resulting in 37.5 DXAs per person year fewer performed in 2012 compared to 2006.

Conclusion-Since July 2009 a significant decline in DXA utilization occurred in a younger postmenopausal commercially insured population. This decline corresponds with a time period of reductions in Medicare DXA reimbursement.

\section{Keywords}

Dual energy x-ray absorptiometry (DXA); Reimbursement; Utilization; Osteoporosis

Bone mineral density measurement using dual energy $\mathrm{x}$-ray absorptiometry (DXA) is the most common method for diagnosing osteoporosis and identifying patients with low bone mass at risk for fractures. ${ }^{(1,2)}$ In 2010, 10.3 million US men and women 250 were estimated to have osteoporosis, with 43.1 million having low bone mass. ${ }^{(3)}$ A 2004 systematic review of studies investigating the use of DXA in patients with fragility fractures reported a testing rate of less than $32 \%$ in 14 of 16 studies referenced, indicating a low rate of testing even in a population at a high risk for fracture. ${ }^{(4)}$

To standardize coverage and costs of DXA screening nationwide, the 1998 Bone Mass Measurement Act provided Medicare Part B coverage of DXAs up to every two years for beneficiaries with specific conditions (including all post-menopausal women) older than age 65. ${ }^{(5)}$ However to control Medicare expenditures, reimbursement for DXA and other outpatient imaging services were reduced under the Deficit Reduction Act of 2005. The act reduced DXA reimbursement to $\$ 55$ in 2010, well below the estimated median cost of $\$ 134.13$ in 2007 for outpatient DXAs and significantly less than the mean reimbursement of $\$ 139$ in 2006. ${ }^{(6,7)}$ On July 1, 2009 the Centers of Medicare \& Medicaid Services (CMS) in a yearly revision to the Physician Fee Schedule (CMS-1413-P) reduced reimbursement for resource value units associated with DXA which reduced reimbursement to $\$ 45$ beginning in 2010. ${ }^{(8,9)}$ The CMS-1413 changes were implemented, however their effect was not felt in 2010 or 2011 as the Affordable Care Act (ACA) temporarily increased reimbursement for these two years. ${ }^{(10)}$ But on March 1, 2012 the full reimbursement cuts were actualized resulting in a reported mean reimbursement of $\$ 56$ per DXA test. ${ }^{(10)}$

Changes in DXA reimbursement have had the potential to significantly affect utilization in both the Medicare and commercially insured population. Although reimbursement reductions targeted the Medicare (aged 65 ) population, fewer outpatient centers were reported to offer DXAs by 2009, which could also affect DXA utilization in the commercially insured population. ${ }^{(11)}$ Studies of DXA utilization through 2011 have been undertaken in both the Medicare and commercially insured population with differing results. In the Medicare fee-for-service population DXA utilization has been shown to increase from 2002 until 2008 or 2009 depending on study before small declines in 2010 and 2011. ${ }^{(6,11,12)}$ In patients with commercial insurance, decreases between 2005 and 2008 were reported for patients with Medicare Supplementary Insurance and increases between 
2000 and 2009 for commercially insured patients less than age 64. ${ }^{(13,14)}$ Overall, and based on these reports that describe a diverse population including Medicare and/or commercially insured patients and covering time periods wherein reimbursement cuts were being phased in, DXA utilization appears to have slowed and in some reports slightly declined.

To better describe DXA utilization from the initial proposed reduction in 2006 to the actual reduction in 2012, as well as update utilization estimates through 2012 we undertook this study. We examined the use of DXA by commercially insured younger postmenopausal women (aged 50-64) from 2006 through 2012 to determine how DXA utilization had changed during this period. Also we investigated the longitudinal use of DXA to determine if a specific event or time period where DXA utilization significantly changed.

\section{Methods}

We studied a cohort of women aged 50-64 between January 2006 and December 2012 in Truven Health Analytics MarketScan ${ }^{\circledR}$ Commercial Claims database. The Marketscan ${ }^{\circledR}$ database is a collection of US employer-sponsored health plans, including de-identified individual-level clinical utilization, expenditures, and enrollment across inpatient, outpatient, prescription drug, and carve-out services with 60 million covered lives in 2011. ${ }^{(15)}$ The University of North Carolina Institutional Review Board determined that this study was exempt.

Women aged 50-64 were selected for this study as they likely comprise the postmenopausal population younger than Medicare age who would utilize DXA. DXAs were identified as claims with HCPCS (Healthcare Common Procedure Coding System) codes 70675 or 77080. When two DXA claims were present within 15 days of each other (e.g. one for performing the service, and the other for physician interpretation), we aggregated the claims as a single DXA claim, based on prior methodology. ${ }^{(1)}$ This study intended to measure overall DXA utilization, as such we did not attempt to differentiate between initial and serial testing. Women were stratified into 5 year age groups $(50-54,55-59,60-64)$ based on age from the monthly enrollment file. Utilization was grouped by either month or year as specified, women could add multiple DXAs to the numerator and were only required to be aged 50 to 64 and enrolled in a health plan for at least one day in the time period to be included in the denominator based on previously published methodology. ${ }^{(16)}$ We calculated rates of DXA utilization per 1,000 person years (PY) both in aggregate and by 5 year age groups, by month and year as specified.

Given that utilization changes were expected during the study period based on prior research, we used visual inspection to determine the inflection point in the trend of DXAs. From this inspection, July 2009 was determined as the point at which utilization was altered and this point was used in our time series models to compare pre- and post-policy reimbursements effects on DXA utilization. This point also coincides with a CMS policy (CMS-1413-P) which further reduced 2012 reimbursement. ${ }^{(8)}$

To evaluate the utilization change before and after July 2009 we used segmented linear regression. ${ }^{(17,18)}$ The regression models included a constant term (rate in January 2006), 
linear time trend (ranging from 1-84 for each month of analyzed data), and a linear time trend to assess the changes in slope of the trend line after July 2009 (ranging from 1 [July 2009] to 41 [December 2012]). Statistical significance was set at $\mathrm{p}<0.05$ a priori. The full regression was modeled as:

$$
Y_{t}=\beta_{0}+\beta_{1} T I M E+\beta_{2} A F T E R
$$

Autoregressive integrated moving average (ARIMA) models were used to control for autocorrelations and seasonality in DXA utilization and conducted using SAS 9.4 (SAS Institute, Cary, NC). Our final model did not include a one-time disruption point at July 2009 as we felt that the change in utilization would be gradual rather than abrupt. When the disruption point was used in the models it was not significant and our results were similar.

\section{Results}

Seasonally adjusted age stratified DXA utilization per 1,000 PY by month are presented as the Figure. In aggregate 451,656 DXAs were performed in 2006, yielding a rate of 144.1 scans per 1,000 PY, increasing to 148.8 DXAs per 1,000 PY in 2009 before a decline to 109.7 DXAs per 1,000 PY or 667,982 scans in 2012. Age stratified segmented regression results are presented as the Table. DXA utilization in January 2006 for the aggregate population was 142.1 DXAs (95\% confidence interval [95\% CI]: 137.66, 146.92) per 1,000 PY. If CMS-1413-P had not occurred, yearly DXA utilization was estimated to increase by 2.24 (95\% CI: $0.18,4.31)$ per $1,000 \mathrm{PY}$, however the yearly utilization trend changed to -12.98 (95\% CI: $-17.19,-8.78$ ) DXAs per 1,000 PY in July 2009. Presumably without CMS-1413-P we would have expected a rate of 157.98 DXAs per 1,000 PY in December 2012, however we observed 104.70 DXAs per 1,000 PY a decrease of 53.29 DXAs per $1,000 \mathrm{PY}$. These significant reductions were found in aggregate and all three age groups, with women aged 60-64 seeing the largest decline.

\section{Discussion}

In a commercially insured population of women aged 50-64 we report a decrease of 35 DXAs per 1,000 PY by year between 2006 and 2012. DXAs were predicted by year to increase by 2.24 DXAs per 1,000 PY for the duration of the study, however after further reductions in CMS-1413-P (July 2009) utilization decreased by 12.98 DXAs per 1,000 PY. Overall, DXA utilization declined between 2006 and 2012.

Our yearly results are similar to those of commercially insured and Medicare beneficiaries through 2010. Based on the same database we report a similar magnitude of increase in DXA utilization between 2006 and 2009 to O'Malley et al. ${ }^{(14)}$ Our analysis is of women aged 50 to 64, however our yearly estimates follow a similar patterns to estimates of both Medicare and Medicare supplemental insurance patients. Between 2008 and 2009 where O'Malley et al and King et al reported decreases in DXA utilization, Zhang et al reported no change, and we report an increase in utilization. ${ }^{(6,11,14)} \mathrm{We}$ would speculate that these differences are due to differing at-risk populations and utilization measures. Additionally, our results are not directly comparable to Morden et al as they only report yearly utilization 
for 2008, 2010, and 2011 and are based on Medicare patients; though their results indicate a decrease of 0.4 DXAs per 100 beneficiaries between 2010 and 2011, which are similar to our results. ${ }^{(12)}$

Although we highlight CMS-1413-P, other rationales for decreasing DXA utilization include fewer outpatient facilities offering DXAs, lower reimbursement by commercial insurers, reduced clinical rationale due to clinical guideline recommendations against routine screening in women aged 50 to 64, fewer inappropriate short interval repeat DXAs, as well as the possibility that patients may have indicated they would decline treatment regardless of the findings of the DXA. ${ }^{(11,12)}$ It is also possible that the change in utilization after CMS-1413P may indicate that at a final rate of $\sim \$ 45$, DXAs were no longer cost effective and centers ceased offering them. Overall our results suggest that Medicare DXA reimbursement changes may have indirect consequences on commercially insured women ages 50-64 even prior to Medicare eligibility.

Our study represents an updated estimate of DXA utilization through 2012, however there are limitations. Our results are based on a commercially insured population aged 50 to 64 who may not be directly comparable to Medicare beneficiaries. We compare our results to other studies with differing methodologies, data sources, and study populations which may not be directly comparable. Although we highlight the proposal of CMS-1413-P (July 2009) as the point where utilization was altered it is likely that other factors as well as other policies affected the DXA utilization up to and after this point. We discuss Medicare reducing reimbursement for DXAs, which likely affected the reimbursement rates for commercial insurance carriers which also may have affected utilization. Reimbursement rates for commercial insurers are not publicly available, as such are not discussed in this report. The strengths of our analysis include representation of 7 years of data in a large commercially insured population as well as segmented regression analysis indicating a time period of significant change in utilization after further reimbursement reduction.

As prior research of DXA utilization in a commercial population is only available through 2009, our study presents updated information on the utilization of DXAs during Medicare rate reductions. Also we were able to find and support a specific policy during reimbursement changes which was associated with further reduced utilization. Though legislative attempts have been made to increase DXA reimbursement rates, the 2014 reimbursement rate for outpatient DXAs is less than $\$ 60$, significantly less than before the reimbursement cuts and likely below the current cost of performing the test. Reduced DXA utilization may be associated with an increase in preventable fractures. As such, follow up studies should examine DXA utilization in additional populations and the influence of reductions in DXA reimbursement rates on clinical outcomes and associated changes in healthcare expenditures.

\section{Acknowledgement}

The database infrastructure used for this project was funded by the Department of Epidemiology, UNC Gillings School of Global Public Health; the Cecil G. Sheps Center for Health Services Research, UNC; the CER Strategic Initiative of UNC's Clinical Translational Science Award (1 ULI RR025747); and the UNC School of Medicine.

Funding Sources: None 


\section{REFERENCES}

1. World Health Organization. Assessment of fracture risk and its application to screening for postmenopausal osteoporosis. Report of a WHO Study Group. World Health Organ Tech Rep Ser. 1994; 843:1-129. [PubMed: 7941614]

2. Siris ES, Adler R, Bilezikian J, Bolognese M, Dawson-Hughes B, Favus MJ, Harris ST, Jan de Beur SM, Khosla S, Lane NE, Lindsay R, Nana AD, Orwoll ES, Saag K, Silverman S, Watts NB. The clinical diagnosis of osteoporosis: a position statement from the National Bone Health Alliance Working Group. Osteoporos Int. 2014; 25:1439-1443. [PubMed: 24577348]

3. Wright NC, Looker AC, Saag KG, Curtis JR, Delzell ES, Randall S, Dawson-Hughes B. The Recent Prevalence of Osteoporosis and Low Bone Mass in the United States Based on Bone Mineral Density at the Femoral Neck or Lumbar Spine. J Bone Miner Res. 2014

4. Elliot-Gibson V, Bogoch ER, Jamal SA, Beaton DE. Practice patterns in the diagnosis and treatment of osteoporosis after a fragility fracture: a systematic review. Osteoporosis Int. 2004; 15:767-778.

5. Health Care Financing Administration, Department of Health and Human Services (US). Medicare Program; Medicare Coverage of and Payment for Bone Mass Measurements. Interim final rule with comment period. Fed Regist. 1998; 63:34320-34328. Available from. [PubMed: 10180295]

6. King AB, Fiorentino DM. Medicare payment cuts for osteoporosis testing reduced use despite tests' benefit in reducing fractures. Health Aff (Millwood). 2011; 30:2362-2370. [PubMed: 22147865]

7. Lewin Group Inc. Assessing the costs of performing DXA services in the office-based setting. 2007

8. Centers for Medicare \& Medicaid Services, Department of Health and Human Services (US). Medicare Program; Payment Policies under the Physician Fee Schedule and Other Revisions to Part B for CY 2010. CMS-1413-P. Fed Regist. 2009; 74:33520-33825. Available from: http:// edocket.access.gpo.gov/2009/pdf/E9-15835.pdf.

9. International Society for Clinical Densitometry. Update on DXA in DC CMS Proposes More Cuts. DXA Bill Gains Sponsors. 2009 Available from: http://www.iscd.org/dxa-reimbursemen-tissues/ update-on-dxa-in-dc-cms-proposes-more-cuts-dxa-bill-gains-sponsors/.

10. Boyle AM. Bone density tests strengthen bottom line. Medical Economics. 2012 Available from: http://medicaleconomics.modernmedicine.com/medical-economics/news/modernmedicine/ modern-medicine-feature-articles/bone-density-tests-strengthen.

11. Zhang J, Delzell E, Zhao H, Laster AJ, Saag KG, Kilgore ML, Morrisey MA, Wright NC, Yun H, Curtis JR. Central DXA utilization shifts from office-based to hospital-based settings among medicare beneficiaries in the wake of reimbursement changes. J Bone Miner Res. 2012; 27:858864. [PubMed: 22190195]

12. Morden NE, Schpero WL, Zaha R, Sequist TD, Colla CH. Overuse of short-interval bone densitometry: assessing rates of low-value care. Osteoporos Int. 2014

13. McAdam-Marx C, Unni S, Ye X, Nelson S, Nickman NA. Effect of Medicare reimbursement reduction for imaging services on osteoporosis screening rates. J Am Geriatr Soc. 2012; 60:511516. [PubMed: 22329356]

14. O'Malley CD, Johnston SS, Lenhart G, Cherkowski G, Palmer L, Morgan SL. Trends in dualenergy X-ray absorptiometry in the United States, 2000-2009. J Clin Densitom. 2011; 14:100 107. [PubMed: 21787516]

15. Hansen L, Chang S. White Paper: Health Research Data for the Real World: The Marketscan(R) Databases. 2013

16. Overman RA, Freburger JK, Assimon MM, Li X, Brookhart MA. Observation stays in administrative claims databases: underestimation of hospitalized cases. Pharmacoepidemiol Drug Saf. 2014; 23:902-910. [PubMed: 24866538]

17. Wagner AK, Soumerai SB, Zhang F, Ross-Degnan D. Segmented regression analysis of interrupted time series studies in medication use research. J Clin Pharm Ther. 2002; 27:299-309. [PubMed: 12174032]

18. Farley JF, Dusetzina SB. Medicaid prescription drug utilization and expenditures following Part D. J Health Care Poor Underserved. 2010; 21:715-728. [PubMed: 20453368] 
July 2009

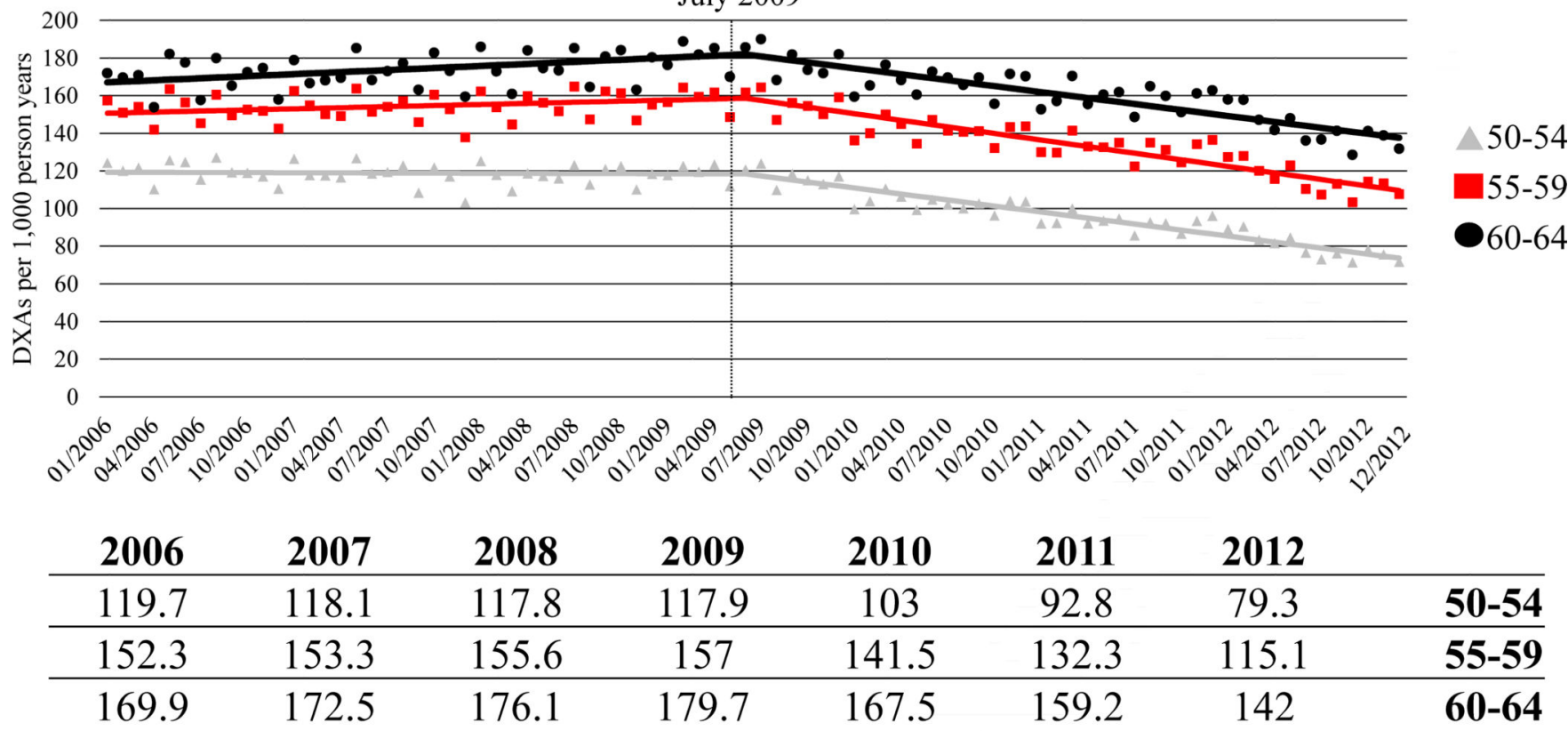

Figure 1.

Dual energy x-ray absorptiometry (DXA) utilization in woman 50 to 64 years of age per 1,000 person years by month (2006-2012) with seasonal adjustment 


\section{Table}

Predicted yearly impact of additional reimbursement reduction in July 2009 from CMS-1413-P on DXA utilization between 2006 and 2012

\begin{tabular}{lcc}
\hline & \multicolumn{2}{c}{ Segmented Regression Results } \\
& Coefficient & 95\% CI \\
\hline Age 50-54 & & \\
Baseline intercept & 119.37 & $(115.85,122.88)$ \\
Time & -0.27 & $(-0.39,-0.14)$ \\
After & -12.51 & $(-12.73,-12.28)$ \\
Change & -12.77 & $(-13.03,-12.51)$ \\
Age 55-59 & & \\
Baseline intercept & 150.53 & $(145.74,155.32)$ \\
Time & 2.31 & $(0.15,4.47)$ \\
After & -16.29 & $(-20.10,-12.48)$ \\
Change & -13.98 & $(-18.36,-9.60)$ \\
Age 60-64 & & \\
Baseline intercept & 166.66 & $(160.44,172.88)$ \\
Time & 4.36 & $(1.54,7.18)$ \\
After & -16.99 & $(-21.97,-12.02)$ \\
Change & -12.63 & $(-18.35,-6.92)$ \\
\hline
\end{tabular}

All coefficients are rate per 1,000 person years per year; Baseline intercept is utilization in January 2006; Time is the linear slope of utilization prior to July 2009; After is the linear slope of utilization after and including July 2009; Change is the change in slope after July 2009 and is calculated as Time + After; All estimates are seasonally adjusted 